\title{
Reconstructing the baryon acoustic oscillations using biased tracers
}

\author{
Jack Birkin, ${ }^{1 \star}$ Baojiu $\mathrm{Li}^{\oplus}{ }^{1}$ Marius Cautun ${ }^{\oplus 1}$ and Yanlong $\mathrm{Shi}^{2,3}$ \\ ${ }^{1}$ Institute for Computational Cosmology, Department of Physics, Durham University, South Road, Durham DH1 3 LE, UK \\ ${ }^{2}$ Department of Astronomy, University of Science and Technology of China, Hefei 230026, Anhui, China \\ ${ }^{3}$ Department of Physics, California Institute of Technology, Pasadena, CA 91125, USA
}

Accepted 2018 December 4. Received 2018 November 28; in original form 2018 September 29

\begin{abstract}
The reconstruction of the initial conditions of the Universe is an important topic in cosmology, particularly in the context of sharpening the measurement of the baryon acoustic oscillation (BAO) peak. Non-linear reconstruction algorithms developed in recent years, when applied to late-time matter fields, can recover to a substantial degree the initial density distribution, however, when applied to sparse tracers of the matter field, the performance is poorer. In this paper, we apply the Shi et al. non-linear reconstruction method to biased tracers in order to establish what factors affect the reconstruction performance. We find that grid resolution, tracer number density, and mass assignment scheme all have a significant impact on the performance of our reconstruction method, with triangular-shaped-cloud mass assignment and a grid resolution of $\sim 1-2 h^{-1}$ Mpc being the optimal choice. We also show that our method can be easily adapted to include generic tracer biases up to quadratic order in the reconstruction formalism. Applying the reconstruction to halo and galaxy samples with a range of tracer number densities, we find that the linear bias is by far the most important bias term, while including non-local and non-linear biases only leads to marginal improvements on the reconstruction performance. Overall, including bias in the reconstruction substantially improves the recovery of BAO wiggles, down to $k \sim 0.25 \mathrm{~h} \mathrm{Mpc}^{-1}$ for tracer number densities between $2 \times 10^{-4}$ and $2 \times 10^{-3}\left(h^{-1} \mathrm{Mpc}\right)^{-3}$.
\end{abstract}

Key words: cosmological parameters - distance scale-large-scale structure of Universe.

\section{INTRODUCTION}

The measurement of distances on large scales is one of the biggest cosmological challenges, with crucial implications for our understanding of the Universe. Distance measurement techniques usually rely on observables which can be predicted theoretically, such as the peak luminosity of a Type Ia supernova light curve, which can be used as a 'standard candle', the Tully-Fisher relation which links the angular velocity of a spiral galaxy with its intrinsic luminosity, or the relationship between the pulsation period of a Cepheid variable and its luminosity. In this work, we are concerned with the baryon acoustic oscillations (BAO) which result from the propagation of sound waves in the baryon-photon fluid prior to recombination that imprints a characteristic length-scale on the large-scale structure of the Universe (Cole et al. 2005; Eisenstein et al. 2005), providing us with a so-called standard ruler that can be used to measure (angular diameter) distances. In the current standard cosmological model, this primordial baryon-photon fluid is highly homogeneous, with tiny density fluctuations. Overdense regions are subject to a higher pressure, causing the fluid to propagate outwards from their centres. These 'ripples' propagate at speeds of order $c$ until recombination, when the photons decouple from the baryons, leaving a shell of baryonic matter with a radius determined by the distance travelled since recombination. Since the Universe contains many of these small overdensities, these shells overlap and interfere, and the result is that matter clusters with a characteristic scale - the BAO scale. Statistically, the BAO manifests as a peak in the matter correlation function, $\xi(r)$, at $r \sim 100 h^{-1} \mathrm{Mpc}$, or as a series of oscillations in the matter power spectrum $P(k)$, which is the Fourier transform of the correlation function (Eisenstein et al. 2007). Large-scale surveys of the past, present and future [e.g. SDSS (Alam et al. 2017), LSST (Ivezić et al. 2008), EUCLID (Laureijs et al. 2011), and DESI (Aghamousa et al. 2016)] map the distribution of matter on large scales, allowing us to measure the angular diameter distance $d_{\mathrm{A}}$ and the Hubble parameter $H$ as a function of redshift and thus map the cosmic expansion history. This can be particularly effective when combined with data from type Ia supernovae and the cosmic microwave background (see e.g. Aubourg et al. 2015).

As the number of large-scale galaxy surveys grows, so does our ability to map the Universe to higher redshifts and measure the size 
of the BAO features to high precision. However, as the majority of our observations are at a relatively low redshift (as an example, DESI will target quasars up to $z \sim 3.5$ for Ly- $\alpha$ forest absorption; Aghamousa et al. 2016), the BAO peak in the correlation function is weakened and broadened due to non-linear structure formation. As a result, measurements of the BAO scale are less precise and the constraints that we can place on our cosmological model are weaker (Wang et al. 2017).

A common solution to this problem is to 'reverse' the evolution of the Universe and recover the distribution of matter at early times, before non-linear evolution weakened the BAO signal. This process, known as 'reconstruction', dates back long before the discovery of the BAO to the work of Peebles (1989), who attempted to predict the trajectories of Local Group galaxies by applying the principle of least action. Weinberg (1992) proposed the 'Gaussianization' method for reconstruction, which is centred on the assumption that gravitational evolution preserves the rank order of the initial density field. Croft \& Gaztanaga (1997) introduced the Path Interchange Zel'dovich Approximation (PIZA) method, assuming that the initial conditions are homogeneous and swapping pairs of particles in the final distribution until the total action in the evolution between the initial and final states is minimized. Recently, forward reconstruction models of the initial conditions have gained a lot of attention (e.g. Kitaura \& Enßlin 2008; Jasche \& Wandelt 2013; Wang et al. 2014; Lavaux 2016). These employ efficient Monte Carlo sampling of the initial power spectrum and phases, which is then non-linearly evolved to low redshift and compared against observations. While such methods can recover the initial conditions down to scales of a few Mpc, they come at the expense of a large computational cost and complex modelling of bias and redshift-space distortions (e.g. see Jasche \& Lavaux 2018).

It was first shown by Eisenstein et al. (2007) that the weakening of the BAO signal is reversible, by suggesting that one can use linear theory to determine the velocity field from the density field, and subsequently reverse the gravitational flow of objects to (almost) recover their initial positions. Even with this relatively simple argument, Eisenstein et al. have shown that the reconstruction procedure can considerably enhance the BAO peak in the correlation function, or equivalently the oscillations in the power spectrum. Padmanabhan et al. (2012) provided the first application of reconstruction to survey data, finding a $\sim 50$ per cent reduction in the uncertainty associated to the BAO scale measurement in the SDSS Data Release 7 (see also Aubourg et al. 2015; Alam et al. 2017, for more recent examples).

The method mentioned above makes use of the Zel'dovich approximation which is accurate down to quasi-linear scales. More recently proposed techniques, including our own (see Shi, Cautun \& Li 2018), extend into the non-linear regime and can therefore recover information from the initial conditions on scales of several Mpc. For example, a method which is closely related to ours is the Monge-Ampeŕe-Kantorovich technique of Frisch et al. (2002), Brenier et al. (2003), and Mohayaee et al. (2006). These works presented and subsequently built on the idea that reconstruction can be treated as an example of the optimal mass transportation problem. We will see in Section 2 that our method begins with the same basic principles and assumptions. More recent non-linear methods include, but are not limited to: the non-linear isobaric reconstruction technique of Zhu et al. (2017, see also Wang et al. 2017), the iterative technique described in Schmittfull, Baldauf \& Zaldarriaga (2017) and the multigrid relaxation method proposed by Shi et al. (2018), the latter of which this work will build on. All of the aforementioned methods have been shown to be capable of recovering the initial conditions on intermediate to non-linear scales when applied to a late-time matter field, and, in the case of the Zhu et al. method, a late-time halo field (Yu, Zhu \& Pen 2017). For example, Wang et al. (2017) showed that isobaric reconstruction could significantly recover the BAO signal from the matter field.

When a reconstruction method is applied to a tracer field, such as a halo or a galaxy field, an additional complication is the biasing between the tracer and underlying matter fields. Dark matter haloes and galaxies, for example, are known to be biased tracers, i.e. their density fields are different from the matter density field. Reconstruction directly from the former, therefore, can lead to errors in the recovery of the initial matter distribution and hence the position and width of the BAO peaks. This issue has been discussed in, e.g. Wang \& Pen (2018) which shows the non-negligible effect of halo bias on the reconstruction of BAO wiggles.

In this paper, we extend the reconstruction method of Shi et al. (2018) to accommodate biased tracers and develop it further to include up to quadratic-order bias schemes. We then investigate how including these bias terms impacts on the reconstruction performance and results. Furthermore, we also study the effect of grid size, mass assignment scheme, and tracer number density on the reconstruction performance. We do so for both halo and galaxy distributions with varying number densities.

The paper is organized as follows: in Section 2, we give a brief review of the Shi et al. (2018) reconstruction method and describe the extension for including biased tracers. In Section 3, we detail the simulations used, along with the halo and galaxy fields used in this work. Section 4 contains the main results, including tests of the impacts of a number of factors that can physically or numerically affect reconstruction performance, and the effects of including tracer biases up to the quadratic order. We then show how our biased reconstruction method can help improve the measurements of BAO wiggles from the tracer power spectra. Finally, Section 5 presents a summary of the findings of this paper, conclusions, and discussions of possible future work.

\section{RECONSTRUCTION METHOD}

\subsection{The reconstruction equation}

We assume that the initial Lagrangian position $\boldsymbol{q}$ of a particle can be mapped to its final Eulerian position $\boldsymbol{x}$ by the gradient of a 'displacement potential' $\Theta$, i.e.

$\boldsymbol{q}=\nabla_{x} \Theta(\boldsymbol{x})$.

This is valid on large scales where stream crossing has not occurred. ${ }^{1}$ Note that equation (1) also assumes that there is no curl component in the relation between $\boldsymbol{q}$ and $\boldsymbol{x}$. The absence of stream crossing also implies mass conservation in a given volume element:

$\rho(\boldsymbol{x}) \mathrm{d}^{3} \boldsymbol{x}=\rho(\boldsymbol{q}) \mathrm{d}^{3} \boldsymbol{q} \approx \bar{\rho} \mathrm{d}^{3} \boldsymbol{q}$,

where $\mathrm{d}^{3} \boldsymbol{q}$ and $\mathrm{d}^{3} \boldsymbol{x}$ are the volume elements at the initial and final times, respectively, and $\rho(\boldsymbol{q})$ and $\rho(\boldsymbol{x})$ are the densities of the corresponding volume elements. The Universe is almost homogeneous at

\footnotetext{
${ }^{1}$ Note that, due to the hierarchical nature of structure formation in $\Lambda \mathrm{CDM}$ cosmology, stream crossing, i.e. particles crossing the trajectories of each other, is inevitable on small enough scales. Therefore, the assumption of no stream crossing is good only on large enough scales. We shall see later that this means that the reconstruction method is less accurate on smaller scales.
} 
early times, however, and we can therefore assume that $\rho(\boldsymbol{q}) \approx \bar{\rho}$, where $\bar{\rho}$ is the mean matter density.

Using equation (1), equation (2) can be rearranged to obtain

$\operatorname{det}\left[\nabla^{i} \nabla_{j} \Theta(\boldsymbol{x})\right]=\frac{\rho(\boldsymbol{x})}{\bar{\rho}} \equiv 1+\delta(\boldsymbol{x})$,

where $i, j=1,2$, and 3 represent the three Cartesian coordinates and $\delta$ is the density contrast. The left-hand side of equation (3) represents a Jacobian matrix comprising the derivatives of the three components of $\boldsymbol{q}$ with respect to the three components of $\boldsymbol{x}$. Frisch et al. (2002) found the solution to equation (3) by treating reconstruction as an 'optimization problem' and finding the arrangement of particles which minimizes a 'cost function'. Similar to the PIZA method, an algorithm is used to swap particles in the final distribution until the optimal arrangement is obtained.

In this work, we follow the new and efficient method developed in Shi et al. (2018), and recast equation (3) into a non-linear elliptical partial differential equation (PDE) which can be solved numerically. The result is

$$
\begin{aligned}
\frac{1}{6}\left(\nabla^{2} \Theta\right)^{3} & -\frac{1}{2} \nabla^{i} \nabla_{j} \Theta \nabla^{j} \nabla_{i} \Theta \nabla^{2} \Theta+\frac{1}{3} \nabla^{i} \nabla_{j} \Theta \nabla^{j} \nabla_{k} \Theta \nabla^{k} \nabla_{i} \Theta \\
& =\frac{\rho(\boldsymbol{x})}{\bar{\rho}},
\end{aligned}
$$

where we have used the Einstein summation convention. We shall apply the multigrid relaxation technique to solve equation (4) for $\Theta$, but for numerical implementation, it is essential to split $\nabla^{i} \nabla_{j} \Theta$ into a diagonal part and a traceless part (see Shi et al. 2018 for a more detailed description of the numerical algorithm) as follows

$\nabla^{i} \nabla_{j} \Theta \equiv \frac{1}{3} \delta_{i j} \nabla^{2} \Theta+\bar{\nabla}^{i} \bar{\nabla}_{j} \Theta$,

which can be regarded as a definition of the barred derivative $\bar{\nabla}^{i}$. Inserting equation (5) into equation (4) gives

$$
\begin{aligned}
\left(\nabla^{2} \Theta\right)^{3} & -\frac{9}{2} \bar{\nabla}^{i} \bar{\nabla}_{j} \Theta \bar{\nabla}^{j} \bar{\nabla}_{i} \Theta \nabla^{2} \Theta+9 \bar{\nabla}^{i} \bar{\nabla}_{j} \Theta \bar{\nabla}^{j} \bar{\nabla}_{k} \Theta \bar{\nabla}^{k} \bar{\nabla}_{i} \Theta \\
& -27(1+\delta)=0,
\end{aligned}
$$

which we will refer to as the reconstruction equation from now on.

In Shi et al. (2018), this method was studied in the context of reconstruction from a late-time matter density field, where it was shown to be capable of recovering the sharpness of the first five BAO peaks. However, cosmological observations do not usually provide us with the 3D matter density fields, but instead catalogues of tracers of the large-scale structure, such as galaxies, clusters, quasars or $21 \mathrm{~cm}$ intensities. These tracers are biased, i.e. $\delta_{\text {tracer }}(\boldsymbol{x}) \equiv$ $n_{\text {tracer }}(\boldsymbol{x}) / \bar{n}_{\text {tracer }}-1$, where $n_{\text {tracer }}(\boldsymbol{x})$ is the number density of the tracer type at $\boldsymbol{x}$ and $\bar{n}_{\text {tracer }}$ is its mean value, is generally not equal to the matter density contrast $\delta(\boldsymbol{x}) \equiv \rho(\boldsymbol{x}) / \bar{\rho}-1$. In the simplest case, a constant linear bias $b_{1}$ applies, where $\delta_{\text {tracer }}=b_{1} \delta$, but this usually works only on very large scales, while in general, the bias effects can be more complicated and include non-linear and non-local terms (Fry \& Gaztanaga 1993; Chan, Scoccimarro \& Sheth 2012, see e.g. Desjacques, Jeong \& Schmidt 2018 for a comprehensive review). Clearly, as the reconstruction algorithm described above requires $\delta(\boldsymbol{x})$, while observations give $\delta_{\text {tracer }}(\boldsymbol{x})$, the bias needs to be included in the reconstruction procedure.

As we shall now see, our method can be naturally extended to include the effects of non-linear and non-local biases. For simplicity, here we consider these bias parameters up to second order, in which case the matter and tracer density contrasts are related by

$\delta_{\mathrm{h}}=b_{1} \delta+\frac{b_{2}}{2} \delta^{2}+\gamma_{2} \mathcal{G}_{2}$, where $b_{1}$ is the linear bias, $b_{2}$ is the quadratic bias, $\gamma_{2}$ is a nonlocal bias parameter, and $\delta_{\mathrm{h}}$ replaces $\delta_{\text {tracer }}$ to make the notation more compact, representing the number density contrast of haloes, although this could be interchanged with any tracer type. The nonlocal bias term in equation (7) can be expressed as (Chan et al. 2012):

$\mathcal{G}_{2}=\nabla^{i} \nabla_{j} \Phi_{v} \nabla^{j} \nabla_{i} \Phi_{v}-\left(\nabla^{2} \Phi_{v}\right)^{2}$,

where $\Phi_{v}$ is the velocity potential, which in the Zel'dovich approximation is related to the displacement field by

$\Psi(\boldsymbol{q})=\boldsymbol{x}(\boldsymbol{q})-\boldsymbol{q}=-\nabla \Phi_{v}$.

As $\Psi$ can be expressed as a derivative of $\Theta, \nabla^{2} \Phi_{v}$ and $\nabla^{i} \nabla_{j} \Phi_{v}$ can be written in terms of second-order derivatives of $\Theta$. This suggests a way to include non-local bias in a slightly modified version of the reconstruction equation, equation (6). To see this, let us note

$\nabla^{i} \nabla_{j} \Phi_{v}=\nabla^{i}\left(q_{j}-x_{j}\right)=\nabla^{i} \nabla_{j} \Theta-\delta^{i}{ }_{j}$,

and

$\nabla^{2} \Phi_{v}=\nabla^{2} \Theta-3$.

Substituting equations (10) and (11) into equation (7) gives

$\delta=\frac{\delta_{\mathrm{h}}}{b_{1}}-\frac{b_{2}}{2 b_{1}^{3}} \delta_{\mathrm{h}}^{2}-\frac{\gamma_{2}}{b_{1}}\left(\nabla^{i} \nabla_{j} \Theta \nabla^{j} \nabla_{i} \Theta+4 \nabla^{2} \Theta-\left(\nabla^{2} \Theta\right)^{2}-6\right)$,

in which the second term on the right-hand side is obtained by approximating $\delta \approx b_{1}^{-1} \delta_{\mathrm{h}}$. One can then replace the $\delta$ in equation (6) using equation (12) to derive a modified reconstruction equation, which is still a PDE for $\Theta$ but which is now sourced by $\delta_{\mathrm{h}}$ (the directly observable quantity) rather than $\delta$. The resulting modified reconstruction equation, which is a more general version of equation (6), is given by

$$
\begin{aligned}
\left(\nabla^{2} \Theta\right)^{3} & -\frac{9}{2} \bar{\nabla}^{i} \bar{\nabla}_{j} \Theta \bar{\nabla}^{j} \bar{\nabla}_{i} \Theta \nabla^{2} \Theta+9 \bar{\nabla}^{i} \bar{\nabla}_{j} \Theta \bar{\nabla}^{j} \bar{\nabla}_{k} \Theta \bar{\nabla}^{k} \bar{\nabla}_{i} \Theta \\
& -27\left[1+\frac{\delta_{\mathrm{h}}}{b_{1}}-\frac{b_{2}}{2 b_{1}^{3}} \delta_{\mathrm{h}}^{2}-\frac{\gamma_{2}}{b_{1}}\left(\nabla^{i} \nabla_{j} \Theta \nabla^{j} \nabla_{i} \Theta+4 \nabla^{2} \Theta\right.\right. \\
& \left.\left.-\left(\nabla^{2} \Theta\right)^{2}-6\right)\right]=0 .
\end{aligned}
$$

This can be applied to any distribution of tracers, and reduces to the standard reconstruction equation in the case of the matter density field (i.e. by setting $b_{1}=1, \gamma_{2}=b_{2}=0$, and $\delta_{\mathrm{h}}=\delta$ ).

\subsection{The numerical algorithm}

We solve for $\Theta$ numerically on a discrete grid, i.e. $\Theta \equiv \Theta_{i, j, k}$ where $i, j$, and $k$ are the indices of cells in the $x$-, $y$-, and $z$-directions respectively. A crucial benefit of the operator splitting in equation (5) is that $\nabla^{2} \Theta$ depends on $\Theta_{i, j, k}$, whereas $\bar{\nabla}^{i} \bar{\nabla}_{j} \Theta$ does not. This allows us to treat equation (6) or (13) as a cubic equation for $\nabla^{2} \Theta$, which can be solved for given $\bar{\nabla}_{i} \bar{\nabla}_{j} \Theta$ and $\delta$ (or $\delta_{\mathrm{h}}$ ). From $\nabla^{2} \Theta$, we can then calculate $\Theta_{i, j, k}$.

It is useful to adjust the form of the reconstruction equations before solving them. Let us take equation (13) as an example here and below. Consider the case of an entirely uniform density field, i.e. $\delta(\boldsymbol{x})=0$. From equation $(3), \operatorname{det}\left[\nabla^{i} \nabla_{j} \Theta(\boldsymbol{x})\right]=1$, so the uniform solution is

$\Theta=\Theta_{0}=\frac{1}{2}\left(x^{2}+y^{2}+z^{2}\right)$, 
and we can define a new variable $\theta$ as the perturbation of $\Theta$ around the uniform solution $\Theta_{0}$, i.e.

$\Theta \equiv \Theta_{0}+\theta$.

It is advantageous to rewrite equation (13) in terms of $\theta$ since our method for solving the PDE is iterative and requires an initial guess for the solution. If we are solving for $\theta$, which represents a perturbation from the uniform solution, then a natural choice for an initial guess is zero. Equation (13) can be recast into the following cubic equation for $\theta$

$a\left(\nabla^{2} \theta+3\right)^{3}+b\left(\nabla^{2} \theta+3\right)^{2}+c\left(\nabla^{2} \theta+3\right)+d=0$,

with coefficients

$a=1$

$b=-\frac{18 \gamma_{2}}{b_{1}}$

$c=\frac{108 \gamma_{2}}{b_{1}}-\frac{9}{2} \bar{\nabla}^{i} \bar{\nabla}_{j} \theta \bar{\nabla}^{j} \bar{\nabla}_{i} \theta$

$d=9 \bar{\nabla}^{i} \bar{\nabla}_{j} \theta \bar{\nabla}^{j} \bar{\nabla}_{k} \theta \bar{\nabla}^{k} \bar{\nabla}_{i} \theta-$

$$
\frac{27}{b_{1}}\left(b_{1}+\delta_{h}-\frac{b_{2}}{2 b_{1}^{2}} \delta_{h}^{2}-\gamma_{2} \bar{\nabla}^{i} \bar{\nabla}_{j} \theta \bar{\nabla}^{j} \bar{\nabla}_{i} \theta+6 \gamma_{2}\right),
$$

In practice, the quantities $\nabla^{2} \theta, c$, and $d$ in equation (16) are calculated on a discretized grid ( $a$ and $b$ are constants), and one should add the subscripts $i, j$, and $k$ to label the coordinate of the cell, but these are omitted here for brevity.

As a cubic equation, equation (16) has multiple analytical solutions, meaning we need a method for establishing which solution is physical. To this end, we define the discriminant as

$\Delta \equiv \frac{q^{2}}{4}+\frac{p^{3}}{27}$

where

$p=\frac{3 a c-b^{2}}{3 a^{2}}$,

$q=\frac{2 b^{3}-9 a b c+27 a^{2} d}{27 a^{3}}$

For $\Delta \geq 0$, the equation has a single real root, which is the physical solution, while for $\Delta<0$, there are three real roots, and the physical one must change continuously as $\Delta$ crosses zero. The physical solution in each case is therefore found to be

$\nabla^{2} \theta=-3+\left[-\frac{q}{2}+\Delta^{\frac{1}{2}}\right]^{\frac{1}{3}}+\left[-\frac{q}{2}-\Delta^{\frac{1}{2}}\right]^{\frac{1}{3}}$ if $\Delta \geq 0$,

$\nabla^{2} \theta=-3-\left(-\frac{p}{3}\right)^{\frac{1}{2}} \cos \left[\frac{1}{3}(\sigma+2 \pi)\right]$ if $\Delta<0$,

where

$\cos (\sigma) \equiv \frac{3 q}{2 p}\left(\frac{-3}{p}\right)^{\frac{1}{2}}$,

and $\sigma$ takes a value between 0 and $\pi$.

Equations (20a) and (20b) are then solved to find $\theta$ using a multigrid Gauss-Seidel technique. As previously mentioned, these two equations are discretized on mesh cells $\left(\theta \rightarrow \theta_{i, j, k}\right)$. As $\theta_{i, j, k}$ is not a continuous function, the spatial derivatives such as $\nabla \theta$ have to be calculated as finite differences, e.g.

$\nabla_{\mathrm{x}} \theta=\frac{1}{2 \ell}\left(\theta_{i+1, j, k}-\theta_{i-1, j, k}\right)$. which represents the $x$-component of the gradient of $\theta$, and where $\ell$ is the side size of a cell which is taken as cubic for simplicity. The finite-difference expression in equation (22) is known to have a second-order accuracy, meaning that the error due to the discretization decreases quadratically as we reduce the cell length $\ell$. We can similarly write finite-difference expressions for higher order derivatives of $\theta$ and their products, but for brevity, these are not listed here, and interested readers can find them in Shi et al. (2018).

Upon discretization, equations (20a) and (20b) can be written as an operator $\mathcal{L}^{\ell}\left[\theta_{i, j, k}\right]$ :

$$
\begin{aligned}
\mathcal{L}^{\ell}\left[\theta_{i, j, k}\right]= & \frac{1}{\ell^{2}}\left(\theta_{i+1, j, k}+\theta_{i-1, j, k}+\theta_{i, j+1, k}+\theta_{i, j-1, k}\right. \\
& \left.+\theta_{i, j, k+1}+\theta_{i, j, k-1}-6 \theta_{i, j, k}\right)-\Sigma_{i, j, k}=0,
\end{aligned}
$$

where $\Sigma_{i, j, k}$ is a discretization of the right-hand side of equation (20a) or (20b), depending on the value of $\Delta$. As mentioned above, the use of the operator splitting ensures that $\Sigma_{i, j, k}$ does not contain $\theta_{i, j, k}$, so that $\mathcal{L}^{\ell}\left[\theta_{i, j, k}\right]$ is effectively a linear operator of $\theta_{i, j, k}$.

The Gauss-Seidel relaxation technique can be used to iteratively update the values of $\theta_{i, j, k}$ :

$\theta_{i, j, k}^{n+1}=\theta_{i, j, k}^{n}-\frac{\mathcal{L}^{\ell}\left[\theta_{i, j, k}^{n}\right]}{\partial \mathcal{L}^{\ell}\left[\theta_{i, j, k}^{n}\right] / \partial \theta_{i, j, k}^{n}}$,

where the superscript $n$ represents the value at the $n$th iteration (remember that the use of $\theta$ instead of $\Theta$ gives us the natural choice of $\theta_{i, j, k}^{0}=0$ as the initial guess for the first iteration). While equation (24) is a general expression for non-linear operators $\mathcal{L}$, because $\mathcal{L}^{\ell}\left[\theta_{i, j, k}\right]$ is a linear operator, one can directly write $\theta_{i, j, k}^{n+1}$ analytically as

$$
\begin{aligned}
\theta_{i, j, k}^{n+1}= & \frac{1}{6}\left(\theta_{i+1, j, k}^{n}+\theta_{i-1, j, k}^{n+1}+\theta_{i, j+1, k}^{n}+\theta_{i, j-1, k}^{n+1}\right. \\
& \left.+\theta_{i, j, k+1}^{n}+\theta_{i, j 1, k-1}^{n+1}\right)-\frac{1}{6} \ell^{2} \Sigma_{i, j, k},
\end{aligned}
$$

where we note that the right-hand side uses a mixture of the $n$th and $(n+1)$ th iteration values of $\theta$ in neighbouring cells of cell $(i, j, k)-$ this is because in the Gauss-Seidel method the relaxation iterations always make use of the most updated values of neighbouring cells.

We define the residual $\varepsilon$ as

$\epsilon \equiv\left[\frac{1}{N^{3}} \sum_{i, j, k=1}^{N}\left(\mathcal{L}^{\ell}\left[\theta_{i, j, k}\right]\right)^{2}\right]^{1 / 2}$,

where $N$ is the number of cells along each axis. Provided the algorithm is stable, $\varepsilon$ decreases as the number of iterations increases. Convergence is deemed to have occurred for $\varepsilon<10^{-8}$, at which point the iterations stop and $\theta$ is outputted along with $\nabla_{x} \theta$. To improve the convergence, we have used the multigrid technique (Press et al. 2007), which employs a hierarchy of coarser meshes to speed up the decrease of $\varepsilon$ (see Shi et al. 2018, for more details).

The method for calculating $\theta$ and $\nabla_{x} \theta$ is incorporated into the ECOSMOG code (see Li et al. 2012), which is based on the publicly available $N$-body simulation code RAMSES (Teyssier 2002). This gives us the values of $\theta(\boldsymbol{x})$ and $\nabla_{x} \theta$ on a uniform $\boldsymbol{x}$-grid, from which we can calculate the corresponding $\boldsymbol{q}(\boldsymbol{x})$ coordinates. Then, the displacement field, $\Psi(\boldsymbol{q})=\boldsymbol{x}-\boldsymbol{q}$, represents a vector defined at an irregular set of points with coordinates $\boldsymbol{q}$ and can be used to calculate the reconstructed initial density field, $\delta_{\mathrm{r}}$, as

$\delta_{\mathrm{r}}=\nabla_{\boldsymbol{q}} \cdot \Psi(\boldsymbol{q})$, 
Table 1. Cosmological parameters and simulation details. The values of the density parameters, $\Omega$, correspond to those at present day.

\begin{tabular}{llcc}
\hline \multicolumn{2}{c}{ Cosmological parameters } & \multicolumn{2}{c}{ Simulation details } \\
Parameter & Value & Parameter & Value \\
\hline$\Omega_{\mathrm{m}}$ & 0.3072 & Particle number & $1024^{3}$ \\
$\Omega_{\mathrm{b}}$ & 0.0481 & Box size & $1 h^{-1} \mathrm{Gpc}$ \\
$\Omega_{\Lambda}$ & 0.6928 & Particle mass & $7.94 \times 10^{10} h^{-1} \mathrm{M}_{\odot}$ \\
$h$ & 0.68 & Refinement & $4,4,4,5,6,7,8,8 \ldots$ \\
$\sigma_{8}$ & 0.8205 & & \\
\hline
\end{tabular}

which we implement using the DTFE (Delaunay Tessellation Field Estimator) code (Cautun \& van de Weygaert 2011; Schaap \& van de Weygaert 2000, see Section 3.2.1 for more details). Note that this calculation is very similar to the use of DTFE to compute the velocity divergence field, for which we have the velocities $\mathbf{v}(\boldsymbol{x})$ (analogous to $\boldsymbol{\Psi}(\boldsymbol{q})$ ) of a set of particles with known $\boldsymbol{x}$-coordinates (analogous to the $\boldsymbol{q}$-coordinates).

\section{SIMULATIONS}

\subsection{Simulation details}

We adopt a $\Lambda \mathrm{CDM}$ cosmology in our simulations. The specifications of the simulations, along with their cosmological parameters, can be found in Table 1. Initial conditions were generated using second-order Lagrangian perturbation theory (2LPT, the 2LPTIC code) (see Scoccimarro 1998) at $z_{\mathrm{i}}=49$, which has been found to be a suitable choice of initial redshift for 2LPT initial conditions (Crocce, Pueblas \& Scoccimarro 2006). We evolve the initial conditions using the RAMSES code, which uses adaptive mesh refinement when solving the Poisson equation, meaning that the simulations begin with a uniform domain grid until the number of particles within a cell exceeds some refinement criterion (see Table 1), at which point the cell is refined to achieve a higher resolution. In our case, this means that a cell will refine itself when it contains four particles, and the resulting cells will refine themselves again when they contain four particles. This pattern will continue using the refinement criterion given in Table 1.

In order to highlight the BAO signal, in what follows we shall compare the matter power spectra from a full simulation with those from a paired no-wiggle simulation, $P_{\mathrm{nw}}$. To generate initial conditions for these two sets of simulations, we calculated the initial matter power spectra with and without the BAO signal using the transfer functions of Eisenstein \& Hu (1998), and used these as the input to 2LPTIC. More information can be found in that work, although we will state here that these functions are appropriate for a high baryon model, which is not what we are using here. However, the objective of this work is not to accurately model the BAO wiggles, but to test to what extent the reconstruction method can recover them. The initial conditions for the paired simulations with and without $\mathrm{BAO}$ wiggles were generated using the same random number seeds to ensure that the corresponding initial density fields have the same random phases and only differ by the BAO features.

\subsection{Tracers of the dark matter field}

In this work, we will test the reconstruction technique when starting from late-time halo density fields, in a similar way to the study of Yu et al. (2017), and late-time galaxy density fields, proceeding to examine the effects of including halo/galaxy bias in the reconstruc- tion. Dark matter haloes are a tracer of the total matter distribution, and can be used as a rough proxy for galaxies in a large-scale survey. As tracers such as galaxies and dark matter haloes generally have much lower number densities than the dark matter particles in an $N$ body simulation, naturally the reconstruction performance will be worse than in Shi et al. (2018). However, understanding the effects of using tracers is important since the application of reconstruction to large-scale survey data involves determining the matter density field from tracers.

\subsubsection{Dark matter haloes}

The dark matter halo catalogues used in this paper are generated using the ROCKSTAR halo finder (Behroozi, Wechsler \& Wu 2013). ROCKSTAR uses a variant of the 3D friends-of-friends method with a modified algorithm that requires a reduced number of calculations and therefore a shorter computation time. We approximate haloes as spherical objects and define their boundary to be at the radius within which their mean mass density is 200 times the critical density $\rho_{\text {crit }}$ of the universe. The halo mass, i.e. the mass contained within this radius, is denoted by $M_{200 c}$. Subhaloes are excluded from our analysis. We apply a mass cut-off, i.e. we ignore haloes with a lower mass than this cut-off, which allows us to tune our halo catalogues to a particular number density. This will be important when comparing halo and galaxy reconstructions (we will use equal number densities for these two tracer types), and when testing reconstruction using different tracer number densities.

We calculate the linear halo bias according to

$b_{1}(r)=\frac{\xi_{\mathrm{hh}}(r)}{\xi_{\mathrm{hm}}(r)}$,

where $\xi_{\mathrm{hh}}(r)$ is the halo autocorrelation function and $\xi_{\mathrm{hm}}(r)$ is the cross-correlation between the halo and the dark matter distributions. Since we have chosen a standard $\Lambda \mathrm{CDM}$ cosmology, the linear bias $b_{1}$ is constant for large scales. $\xi_{\mathrm{hh}}(r)$ and $\xi_{\mathrm{hm}}(r)$ are computed using the Correlation Utilities and Two-Point Estimates (CUTE) code (Alonso 2012). We calculate the large-scale value of $b_{1}$ by using equation (28) to measure $b_{1}(r)$ at different scales, $r$, and then taking the average value in the scale range $10-70 h^{-1} \mathrm{Mpc}$. While this gives a reasonable estimate, in Section 4 , we test several other $b_{1}$ values around the measured value of equation (28).

According to linear perturbation theory, the non-local bias parameter $\gamma_{2}$ can be calculated by (Chan et al. 2012)

$\gamma_{2}=\frac{-2\left(b_{1}-1\right)}{7}$

although we will see that this expression does not work well for both halo and galaxy reconstruction, so we also test different values of $\gamma_{2}$ to see which value gives the best reconstruction performance for a given tracer number density. We do the same for the non-linear bias to quadratic order, $b_{2}$.

We compare different methods of calculating the number density field of dark matter haloes, $n_{\text {halo }}(\boldsymbol{x})$. The first approach consists of the DTFE (Schaap \& van de Weygaert 2000) method implemented in the DTFE code (Cautun \& van de Weygaert 2011), which offers the ability to compute a continuous density field from the positions of discrete tracers. DTFE constructs a Delaunay triangulation that tessellates the entire volume with tetrahedra whose vertices are given by the distribution of tracers, which can be dark matter particles, haloes, or galaxies. The mass of each tracer particle is distributed among the tetrahedra which have that particle as a vertex. Then, to obtain the density on a regular grid, the mass in each 
Table 2. The parameters of the HOD model used to obtain galaxy catalogues. We use three different $M_{\min }$ values to obtain galaxy number densities of 20,7 , and $2 \times 10^{-4}\left(h^{-1} \mathrm{Mpc}\right)^{-3}$, respectively.

\begin{tabular}{lc}
\hline Parameter & Value \\
\hline $\log M_{\min }$ & $11.22,12.30,13.22$ \\
$\log M_{0}$ & 13.077 \\
$\log M_{1}$ & 14.000 \\
$\sigma_{\log M}$ & 0.596 \\
$\alpha$ & 1.0127 \\
\hline
\end{tabular}

tetrahedron is distributed among the grid cells which intersect that tetrahedron. The tessellation is space filling and thus all grid cells contain a non-zero mass and thus a non-zero density. The DTFE method is adaptive to the local tracer distribution: tracer particles in high number density regions typically distribute their mass to a small region around them, while tracers in low number density regions typically distribute their mass up to large distances.

In the second approach, we use the cloud-in-cell (CIC) and triangular-shaped-cloud (TSC) mass assignment schemes to calculate $n_{\text {halo }}$ on the uniform grid used for reconstruction. In three dimensions, the TSC and CIC assignment schemes respectively distribute the mass of a given particle to the 27 and 8 neighbouring cells which overlap with its 'cloud' (Hockney \& Eastwood 1988). For tracers with a low number density, and using a relatively highresolution computational grid for reconstruction, a lot of grid cells will be left with zero density. As we shall see later, this has a nonnegligible impact on the reconstruction result, because TSC and CIC differ significantly from DTFE in low-density regions, with the latter spreading masses into larger spatial regions.

In the results shown below, we do not weight haloes according to their mass; we treat all haloes used for reconstruction as particles of equal mass. We will briefly comment on the tests and results using halo-mass-weighted reconstruction, and possible future development, in the conclusion section.

\subsubsection{Galaxies}

We build galaxy catalogues by populating haloes using the halo occupation distribution (HOD) method (Berlind \& Weinberg 2002; Zheng et al. 2005), which assumes that the probability of a halo hosting one or more galaxies is dependent on the mass of the halo. Specifically,

$$
\begin{aligned}
& \left\langle N_{\text {cen }}(M)\right\rangle=\frac{1}{2}\left[1+\operatorname{erf}\left(\frac{\log M-\log M_{\min }}{\sigma_{\log M}}\right)\right], \\
& \left\langle N_{\text {sat }}(M)\right\rangle=\left\langle N_{\text {cen }}(M)\right\rangle\left(\frac{M-M_{0}}{M_{1}}\right)^{\alpha},
\end{aligned}
$$

as was suggested by Zheng, Coil \& Zehavi (2007). $\left\langle N_{\text {cen }}(M)\right\rangle$ and $\left\langle N_{\text {sat }}(M)\right\rangle$ are the mean numbers of central and satellite galaxies, respectively, and erf is the error function. The number of galaxies within a halo is then a sum of the number of central and satellite galaxies. The model contains five free parameters, with our choices for these parameter values being shown in Table 2 .

In order to directly compare the performance of the reconstruction method for both haloes and galaxies, it is necessary to tune the tracer number density to be the same in each case. Unlike the friendsof-friends method which tells us the mass of each halo, the HOD method does not predict galaxy masses and we cannot obtain a given number density by having a galaxy stellar mass cut. We instead tune the galaxy number density by changing the $M_{\min }$ parameter, where $M_{\min }$ is the minimum mass of haloes which can host a central galaxy.

The galaxy bias can be calculated in the same way as the halo bias, and also remains constant on large scales.

\section{RESULTS AND DISCUSSION}

Fig. 1 shows a visual comparison of the initial and non-linear matter density fields, the non-linear halo field, and the reconstructed density fields from the non-linear dark matter and halo distributions. All density fields are smoothed using a Gaussian filter with $R=2 h^{-1} \mathrm{Mpc}$, with the exception of (1a), which we have left unsmoothed for comparison with (1b). For panels (1a) and (1b), the initial matter density field at $z=49$ has been calculated using TSC mass assignment, and extrapolated to $z=0$ using the $\Lambda$ CDM linear growth factor. In the second and third columns, we show the non-linear matter and halo density fields respectively on the top, with the resulting reconstructed density field on the bottom. In panels (1a), (1b), (2b), and (3b), there are some regions where the density contrast $\delta$ is less than -1 , implying a negative density $\rho$ : for (1a) and (1b) this is simply a result of the fact that these fields are linearly extrapolated versions of the initial density field, which is also true to leading order for the reconstructed density fields in (2b) and (3b). Visually, there is a greater similarity between (1b) and ( $2 b)$ than ( $1 b)$ and ( $3 b)$, which is because the halo field contains less information than the dark matter field, in particular on small scales.

To test the performance of our reconstruction method quantitatively, we define the correlation coefficient between two density fields $\delta_{1}$ and $\delta_{2}$ as

$r_{12}=\frac{\tilde{\delta}_{1} \tilde{\delta}_{2}^{*}+\tilde{\delta}_{1}^{*} \tilde{\delta}_{2}}{2 \sqrt{\tilde{\delta}_{1} \tilde{\delta}_{1}^{*}} \sqrt{\tilde{\delta}_{2} \tilde{\delta}_{2}^{*}}}$,

where a $*$ indicates the complex conjugate, and $\tilde{\delta}$ is the Fourier transform of the density field, $\delta(\boldsymbol{x})$. The correlation coefficient $r_{12}$ describes the similarity between the two density fields. By definition $r_{12}=1$ if the two fields are identical and $r_{12}=0$ if they are completely unrelated. We are interested in the correlation between the initial and final density fields, which we denote with $r_{\text {if }}$, and the correlation between the initial and reconstructed density fields, which we denote with $r_{\text {ir }}$. We expect to find that $r_{\text {if }}$ is closer to 1 on large scales where evolution is linear, with a decline towards 0 on smaller scales where matter has clustered strongly. The performance of the reconstruction method can be quantified by the difference in $r_{\text {if }}$ and $r_{\text {ir }}$, which tells us how much information it has recovered from the initial conditions. As the main aim of this study is to analyse the ability of the reconstruction method to recover the BAO peaks, it is important to observe an improvement on the scales where the first few and most prominent peaks in the power spectrum $P(k)$ occur $\left(0.05 \lesssim k \lesssim 0.3 h \mathrm{Mpc}^{-1}\right)$. To assess quantitatively the reconstruction performance in different scenarios, we define $k_{80}, k_{50}$, and $k_{20}$ to be the wavenumbers at which the corresponding reconstructed density field is 80 per cent, 50 per cent, and 20 per cent correlated with the initial conditions, respectively.

\subsection{Comparison of mass assignment schemes}

Before testing the effects of tracer biases, we first compare the different mass assignment methods described in Section 3.2.1 in order to better understand their impact on reconstruction performance. 


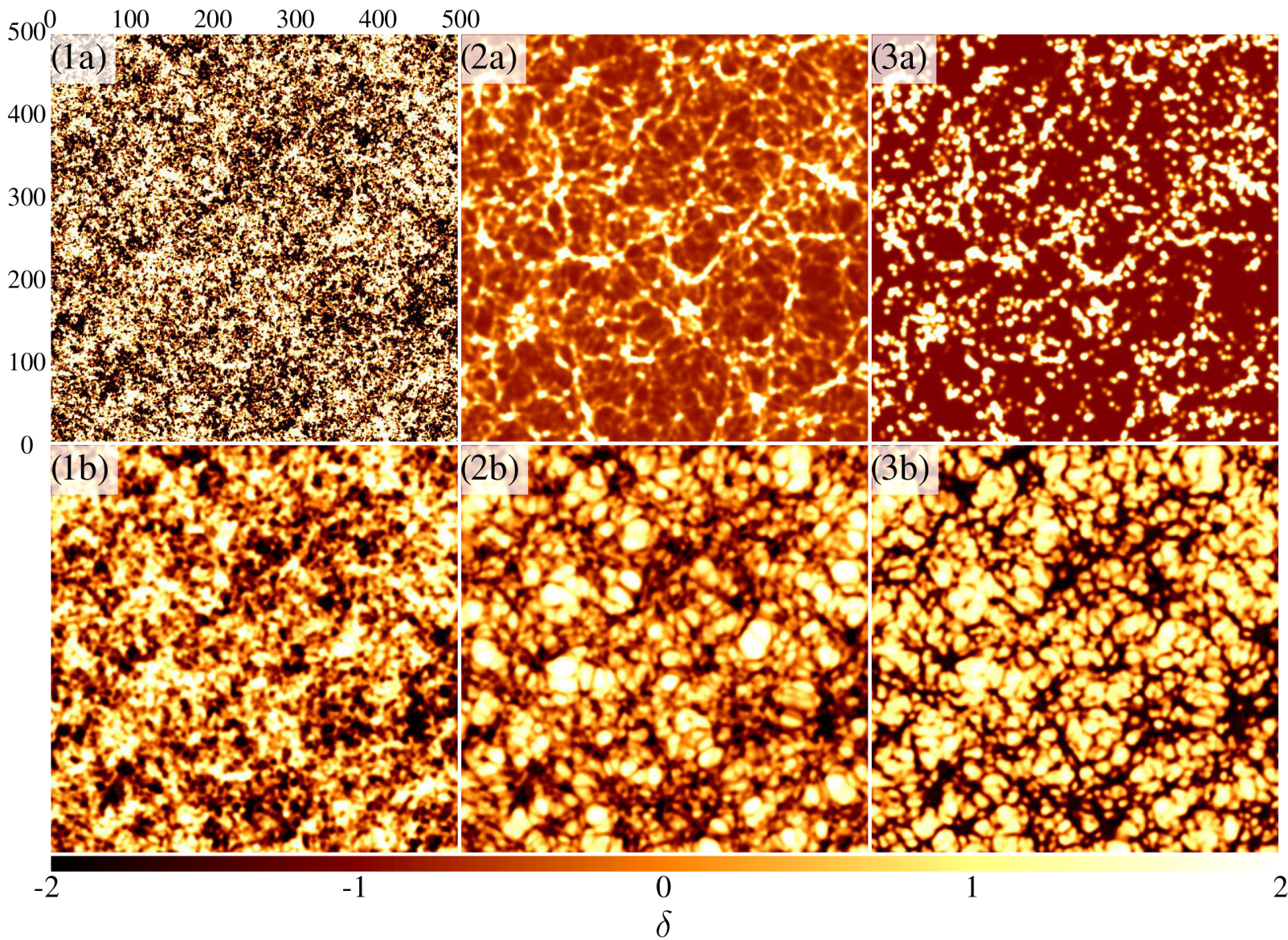

Figure 1. Illustration of the reconstruction results. Each panel shows the same $500 \times 500\left(h^{-1} \mathrm{Mpc}\right)^{2}$ region with $1.0 h^{-1} \mathrm{Mpc}$ thickness of the simulation box. The panels (1a) and (1b) show the initial matter density contrast, $\delta(\boldsymbol{x})$, linearly extrapolated to $z=0$, with (1a) corresponding to the unsmoothed density field, while (1b) corresponds to the field smoothed with a spherical Gaussian filter of size, $R=2 h^{-1}$ Mpc. Panel (2a) shows the non-linearly evolved matter density at $z=0$, and (2b) shows the reconstructed linear density, $\delta_{\mathrm{r}}(\boldsymbol{x})$, from the $z=0$ dark matter distribution. Panel (3a) shows the dark matter halo number density, $\delta_{\mathrm{h}}(\boldsymbol{x})$, at $z=0$, and (3b) shows the reconstructed linear density from the same halo distribution. For ( $\left.3 \mathrm{a}\right)$ and ( $\left.3 \mathrm{~b}\right)$, the halo number density is $2 \times 10^{-3}\left(h^{-1} \mathrm{Mpc}\right)^{-3}$. The density fields in (1b), (2a), (2b), (3a), and (3b) are all smoothed with the same $R=2 h^{-1}$ Mpc Gaussian filter. All six panels use the same colour scheme (see the bottom of the figure) which corresponds to the $\delta$ values shown on a linear scale between $[-2,2]$.

The result is shown in Fig. 2 , with $k_{80}, k_{50}$, and $k_{20}$ values presented in Table 3. From Fig. 2, it is clear that both the CIC and TSC mass assignments perform better than DTFE mass assignment, with improvements found in both $r_{\text {if }}$ and $r_{\text {ir }}$. Regardless of the method used for mass assignment, we find reconstruction to be more effective when using a high tracer number density, as expected. On the other hand, when moving from DTFE to CIC/TSC mass assignment greater improvements are found when the tracer number density is lower, and in the bottom panel, we can see that the non-linear TSC density field is actually more strongly correlated with the initial conditions than the reconstructed density field from DTFE for $k \gtrsim$ $0.2 \mathrm{~h} \mathrm{Mpc}^{-1}$.

The fact that TSC/CIC mass assignment results in a greater improvement over DTFE mass assignment when applied to sparse tracer catalogues is due to the adaptive nature of the DTFE formalism. In DTFE, haloes in low-density regions distribute their mass up to distances many times the mean halo separation, which effectively corresponds to a large-scale smoothing of the density field and inevitably erases information. The largest effective smoothing is for the sparsest halo sample, which is also the one which shows the largest difference in $r_{\text {ir }}$ between the DTFE and the TSC/CIC mass assignments (see Fig. 2). On the other hand, the performances of
TSC and CIC are very similar, with the former slightly better than the latter. Given these tests, in the rest of our analyses we use TSC mass assignment.

\subsection{Comparison of reconstruction grid resolutions}

Here, we investigate the optimal resolution of the regular grid used for the reconstruction procedure. Increasing the grid size, that is reducing the grid spacing, allows us to better recover the initial density on small scales and to reduce discretization errors when solving equation (13) numerically. However, this comes at the price of higher computational resources. There is a physical scale below which structure formation is highly non-linear, representing a physical limit down to which our method can recover the initial density field. This limiting scale can be reached by using a high number density of tracers, such as when applying the reconstruction to the dark matter distribution, however, in the case of halo or galaxy distributions, the limiting scale is likely higher and arises due to the sparsity of the tracer distribution.

We note that varying the grid size employed by our calculation is not the same as varying the smoothing scale used for linear reconstruction methods. Our method is fully non-linear and does not 


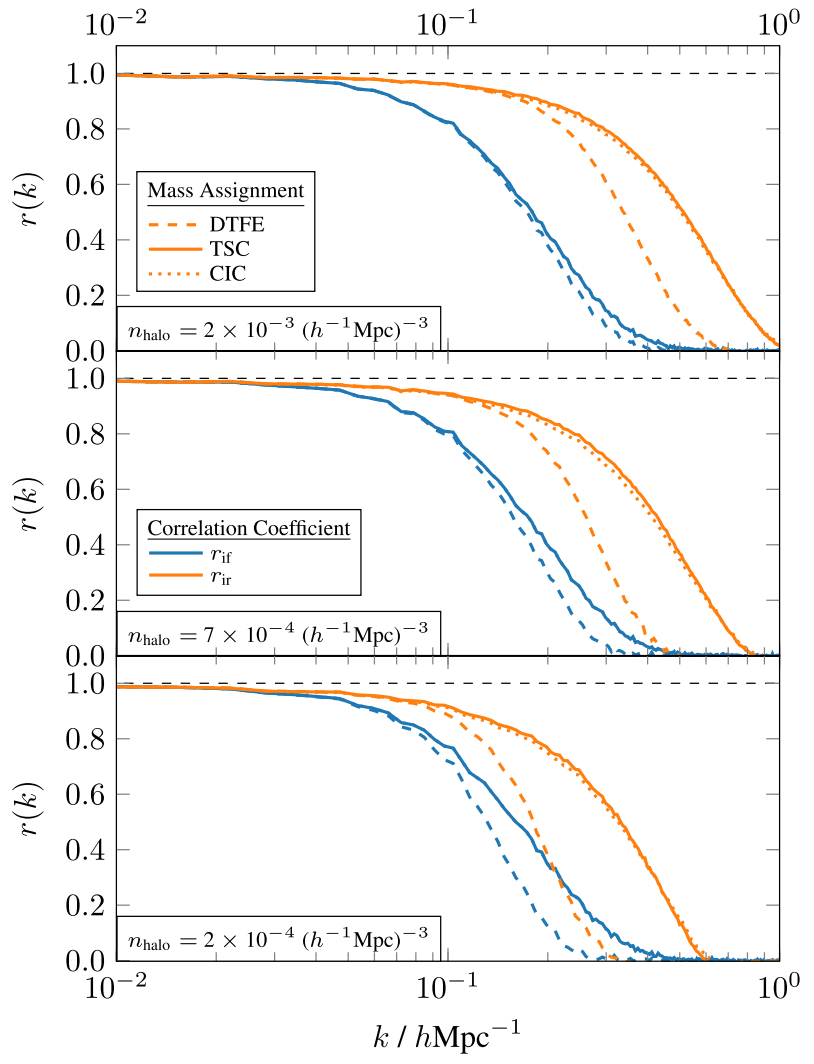

Figure 2. Correlation coefficients between the initial and final density fields, $r_{\text {if }}$ (blue), and between the initial and reconstructed density fields, $r_{\text {ir }}$ (orange). The final and reconstructed density fields were calculated using the halo number density, $\delta_{\mathrm{h}}$, obtained using the DTFE (dashed curves), TSC (solid), and CIC (dotted) mass assignment schemes. The three panels correspond to different halo number density samples, $n_{\text {halo }}=2 \times 10^{-3}$, $7 \times 10^{-4}$, and $2 \times 10^{-4}\left(h^{-1} \mathrm{Mpc}\right)^{-3}$ (from top to bottom).

Table 3. The wavenumbers $k_{80}, k_{50}$ and $k_{20}$ corresponding to the correlation coefficient, $r_{\text {ir }}$, between the initial and reconstructed density fields for two mass assignment schemes, TSC and DTFE, and for three halo samples with different number densities. The wavenumber $k_{\mathrm{f}}$ corresponds to the $k$ value where $r_{\text {ir }}=f$ per cent.

\begin{tabular}{lcccc}
\hline$n_{\text {halo }}$ & Method & $k_{80}$ & $k_{50}$ & $k_{20}$ \\
\hline $2 \times 10^{-3}$ & DTFE & 0.22 & 0.34 & 0.46 \\
& TSC & 0.30 & 0.51 & 0.73 \\
$7 \times 10^{-4}$ & DTFE & 0.18 & 0.26 & 0.34 \\
& TSC & 0.24 & 0.43 & 0.61 \\
$2 \times 10^{-4}$ & DTFE & 0.12 & 0.18 & 0.24 \\
& TSC & 0.18 & 0.33 & 0.47 \\
\hline
\end{tabular}

employ smoothing apart from the effective smoothing caused by assigning particles to the computational grid using, e.g. TSC. Starting from a uniform distribution, our reconstruction finds the minimum displacement field needed to obtain the present-day mass distribution. Using a smaller grid spacing does not affect the largescale modes of the displacement field (although it can reduce discretization errors) and only leads to recovering smaller scale modes. If the scales are small enough to be affected by non-linear structure formation, then the recovered small-scale displacement field is uncorrelated with the original field. Thus, decreasing the grid

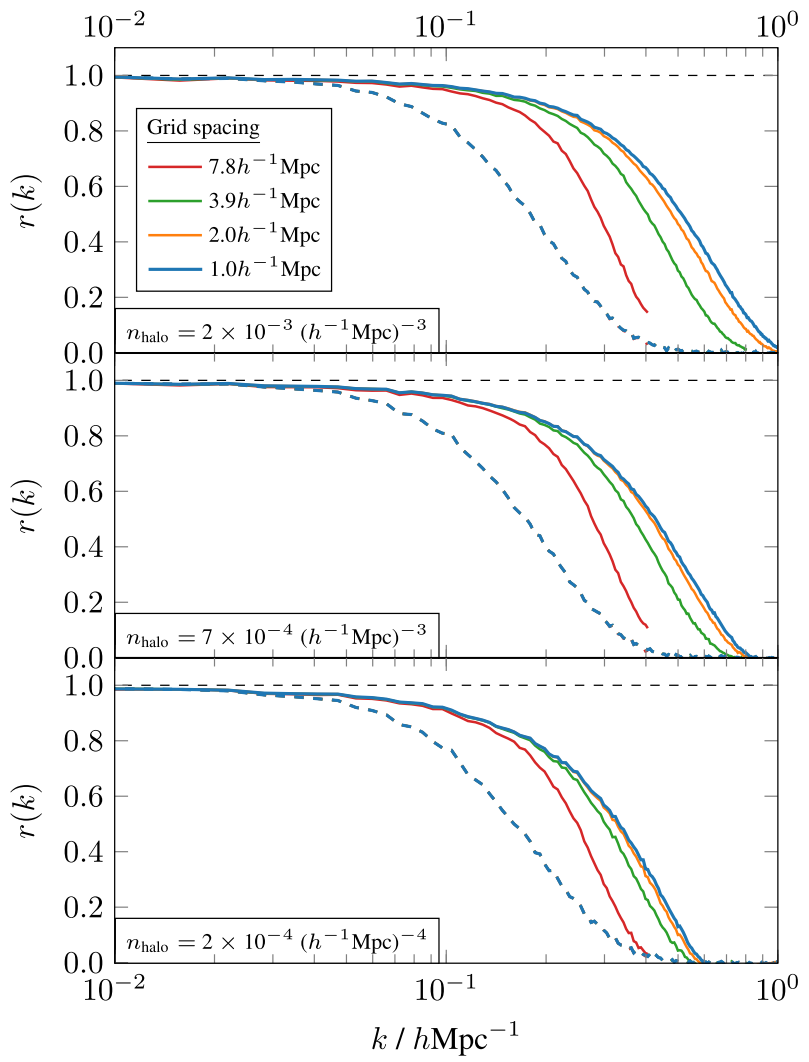

Figure 3. Correlation coefficients between the initial and final density fields $r_{\text {if }}$ (dashed), and between the initial and reconstructed density fields $r_{\text {ir }}$ (solid) for the $n_{\text {halo }}=2 \times 10^{-3}$ (top panel), $7 \times 10^{-4}$ (middle panel), and $2 \times 10^{-4}$ (bottom panel) $\left(h^{-1} \mathrm{Mpc}\right)^{-3}$ halo reconstruction performed using different grid resolutions. The legend shows the reconstruction grid cell spacings. Note that the pink solid lines (the results from grid size $128^{3}$ ) do not reach $r(k)=0$ because for this grid size, the power spectrum is only measured down to a length-scale corresponding to $k=0.4 \mathrm{~h} \mathrm{Mpc}^{-1}$.

size does not affect our reconstruction. In contrast, the performance of linear reconstruction methods, such as the inverse Zel'dovich approach of Eisenstein et al. (2007), is sensitive to the employed smoothing scale. This is because that reconstruction procedure is based on an analytic description of structure formation which is valid only down to quasi-linear scales, with the optimal BAO reconstruction corresponding to a smoothing scale $\sim 10 h^{-1} \mathrm{Mpc}$ (see e.g. Vargas-Magaña et al. 2017).

We employ a grid with uniform spatial resolution, using $\left(2^{n_{l}}\right)^{3}$ cubic cells, where $n_{1}$ is an integer. We test four cases, with $n_{1}=7$, 8,9 , and 10 , respectively. This paper uses a cubic simulation box with $1 h^{-1} \mathrm{Gpc}$ side length, therefore these $n_{1}$ values correspond to a resolution (cubic cell size) of $\ell=7.81,3.91,1.95$, and $0.98 h^{-1} \mathrm{Mpc}$ respectively. Clearly, for larger boxes, larger $n_{1}$ are needed to achieve the same spatial resolution. For simplicity, we consider only halo reconstruction here.

The results are given in Fig. 3. Note that the curves representing the $128^{3}$ grid reconstruction stop at $k \sim 0.4 h \mathrm{Mpc}^{-1}$ because scales smaller than this cannot be sampled on this coarse grid; the same is true for the $256^{3}$ grid, which does not sample scales smaller than $k \sim 0.8 \mathrm{~h} \mathrm{Mpc}^{-1}$. We note that the convergence between different grid resolutions depends sensitively on the tracer number density; for example, grid sizes $\geq 256^{3}$ give similar $k_{80}$ for the case of $n_{\text {halo }}=2 \times 10^{-4}\left(h^{-1} \mathrm{Mpc}\right)^{-3}$, but a $256^{3}$ grid is clearly insufficient for the other two halo number densities. For all three number 
densities, the $512^{3}$ and $1024^{3}$ grids give comparable results, in particular for $k_{80}$ (while for $k_{50}$ and $k_{20}$, the $512^{3}$ grid has not completely converged yet). It is also notable that $r_{\text {if }}$ is independent of the grid size, which was found not to be the case for DTFE mass assignment (not shown here).

Computing time is not an issue for our reconstruction method. For the $1024^{3}$ grid resolution, the reconstruction code takes $\sim 20$ min with 504 CPUs, using 180 GB RAM, and it is much faster for lower grid resolutions. On the other hand, as we shall see below, the grid resolution can be important when including non-linear and non-local halo bias in the reconstruction, because a higher resolution means that $\delta_{\mathrm{h}}$ in equation (13) can become large in cells from highdensity regions, and this will effect the reconstruction performance, resulting in a severe constraint on $b_{2}$, namely $\left|b_{2}\right| \ll 1$. To illustrate the impacts of biased halo reconstruction, therefore, in what follows, we opt to use the $512^{3}$ grid for all our tests. In general, however, where computational resources allow, a higher resolution grid is recommended to make the best of the reconstruction method.

\subsection{Effects of varying tracer bias}

Having fixed the mass assignment scheme and grid resolution, we now move on to analyse the impacts on the reconstruction performance of varying the tracer bias parameters. We start by varying the linear bias, $b_{1}$, then proceed to vary the non-local bias, $\gamma_{2}$, and, finally, the non-linear bias at quadratic order, $b_{2}$, as described in Section 2. More explicitly, we first test a range of values for the linear bias $b_{1}$, fixing $\gamma_{2}=b_{2}=0$, then we fix $b_{1}$ to the best-fitting value and test multiple values of $\gamma_{2}$, then again we fix both $b_{1}$ and $\gamma_{2}$ to their best-fitting values and study the effect of varying $b_{2}$. In this subsection, we focus on the correlation coefficients of the reconstructed density fields, with the impact on the BAO peak recovery being studied in the following subsection.

Figs 4 and 5 show our findings when applying reconstruction to the halo and galaxy distributions, respectively. All panels show the correlation coefficients between the linear matter and non-linear tracer density fields ( $r_{\text {if }}$; dashed), along with those between the linear matter and reconstructed density fields $\left(r_{\text {ir }}\right.$; solid) for a range of bias parameter values for $b_{1}$ (left-hand column), $\gamma_{2}$ (middle column), and $b_{2}$ (right-hand column). As the difference between curves is subtle in many cases, we indicate the chosen 'best' configuration by a thicker curve and a bold value in the legend. The $k_{80}, k_{50}$, and $k_{20}$ values for the highest and lowest tracer number densities are given in Table 4, and we refer to this in our analysis. Given the quite similar behaviour seen in Figs 4 and 5, in the discussion below we focus on the case of halo reconstruction, and comment on galaxy reconstruction when appropriate.

As noted above, a common feature in both $r_{\text {if }}$ and $r_{\text {ir }}$ is the decrease of the correlation coefficient from approximately 1.0 on large scales to 0.0 on small scales, and the rate of this decrease is slower for higher tracer number densities, which contain more accurate information about the underlying dark matter field. In general, reconstruction boosts the correlation coefficient and extends the range of scales over which it is non-zero. We have tested five values of $b_{1}$ for each number density, with the central value being the one calculated using the method outlined in Section 3.2.1. The measured values are $b_{1}=1.2,1.4$, and 1.7 for $n_{\text {halo }}=2 \times 10^{-3}$, $7 \times 10^{-4}$, and $2 \times 10^{-4}\left(h^{-1} \mathrm{Mpc}\right)^{-3}$, respectively. The reconstruction performance is quite sensitive to the value of $b_{1}$ in the highest number density case, though the range of $b_{1} \in[1.2,1.3]$ seems to give very similar results. We chose $b_{1}=1.2$ as our best-fitting value to be fixed when varying $\gamma_{2}$ and $b_{2}$, despite the fact that $b_{1}=1.3$ gives slightly better results on small scales $\left(k>0.6 h \mathrm{Mpc}^{-1}\right)$, as we are more interested in the large scales when aiming to recover the BAO peaks. We choose $b_{1}=1.4$ and 1.7 for $n_{\text {halo }}=7 \times 10^{-4}$ and $2 \times 10^{-4}\left(h^{-1} \mathrm{Mpc}\right)^{-3}$ respectively, noting that the optimal $b_{1}$ value for reconstruction takes the value measured in the simulation for all three number densities. For the two lowest number density samples, the linear bias is not very important and adding \pm 0.2 does not significantly change the reconstruction performance; in the high number density case, however, the result is more sensitive to $b_{1}$ but increasing $b_{1}$ by up to 0.2 from its best-fitting value again has a negligible impact on the correlation coefficient of the reconstructed density field. This is positive news for reconstruction in real observations, where $b_{1}$ is usually not known accurately.

We next employ the optimal linear bias value $b_{1}$ for each number density and repeat the reconstruction process by varying the non-local bias parameter $\gamma_{2}$ in the central column of Figs 4 and 5 . Applying equation (29), we predict $\gamma_{2} \approx-0.06,-0.11$, and -0.20 for the three halo catalogues with decreasing number densities; while trying a range of values for $\gamma_{2}$ in the reconstruction we find $\gamma_{2} \approx-0.05,-0.10$, and -0.30 , respectively, to be the best values. Although not shown here, using the DTFE mass assignment scheme results in an optimal reconstruction when $\gamma_{2} \approx-0.2,-0.3$, and -0.5 for the three corresponding halo number densities. It is noteworthy that the two mass assignment methods lead to different optimal values of the non-local bias, suggesting that the difference in the methods introduces an additional non-physical bias. When using TSC mass assignment, the optimal $\gamma_{2}$ agree more closely with the perturbation theory prediction (Chan et al. 2012), although this agreement is worse in the lowest number density case of halo reconstruction and in galaxy reconstruction. Among the three halo number densities, we find that the greatest improvement in reconstruction performance when including non-local bias is attained for the sparsest sample, where $n_{\text {halo }}=2 \times 10^{-4}\left(h^{-1} \mathrm{Mpc}\right)^{-3}$, for which $\gamma_{2}$ is also the largest. Even in this case, the increase of $k_{80}$ is marginal (0.01), suggesting that including non-local bias in the reconstruction will not substantially improve the recovery of BAO peaks.

The right-hand columns of Figs 4 and 5 show the reconstruction results by fixing $b_{1}$ and $\gamma_{2}$ to their respective bestfitting values for each tracer number density, while varying $b_{2}$ around $b_{2}=0$. For all but the case of halo reconstruction where $n_{\text {halo }}=2 \times 10^{-3}\left(h^{-1} \mathrm{Mpc}\right)^{-3}$, we find that the best-fitting value is $b_{2}=0$, and that any significant deviation from this value would quickly downgrade the performance. As mentioned above, this is because $b_{2}$ enters the reconstruction equation [see equation (13)] through $b_{2} \delta_{\mathrm{h}}^{2}$, so that in high-density regions where $\delta_{\mathrm{h}} \gg 1$, this would lead to a large unphysical contribution to the source of that equation. Physically, the $b_{2}$ bias term has been introduced as a correction which is valid in the regime $\delta \ll 1$, and so should really be used only in the mildly non-linear regime rather than cases where $\delta_{\mathrm{h}}$ $\gg 1$. Indeed, we have explicitly checked that for lower reconstruction grid resolutions, e.g. $128^{3}$ and $256^{3}, b_{2}$ takes larger non-zero best-fitting values; in those cases adding the non-linear bias indeed leads to noticeable improvements in the correlation coefficients $r_{\text {ir }}$, but at the price that $r_{\text {ir }}$ for $b_{2}=0$ is generally much poorer than the $512^{3}$ grid case to start with (cf. Fig. 3). Therefore, at least for the method to model non-linear bias above, using a high-resolution reconstruction grid removes the necessity or appropriateness to include $b_{2}$. More complicated treatments, e.g. which first smooth the tracer number density field before doing the reconstruction, might 


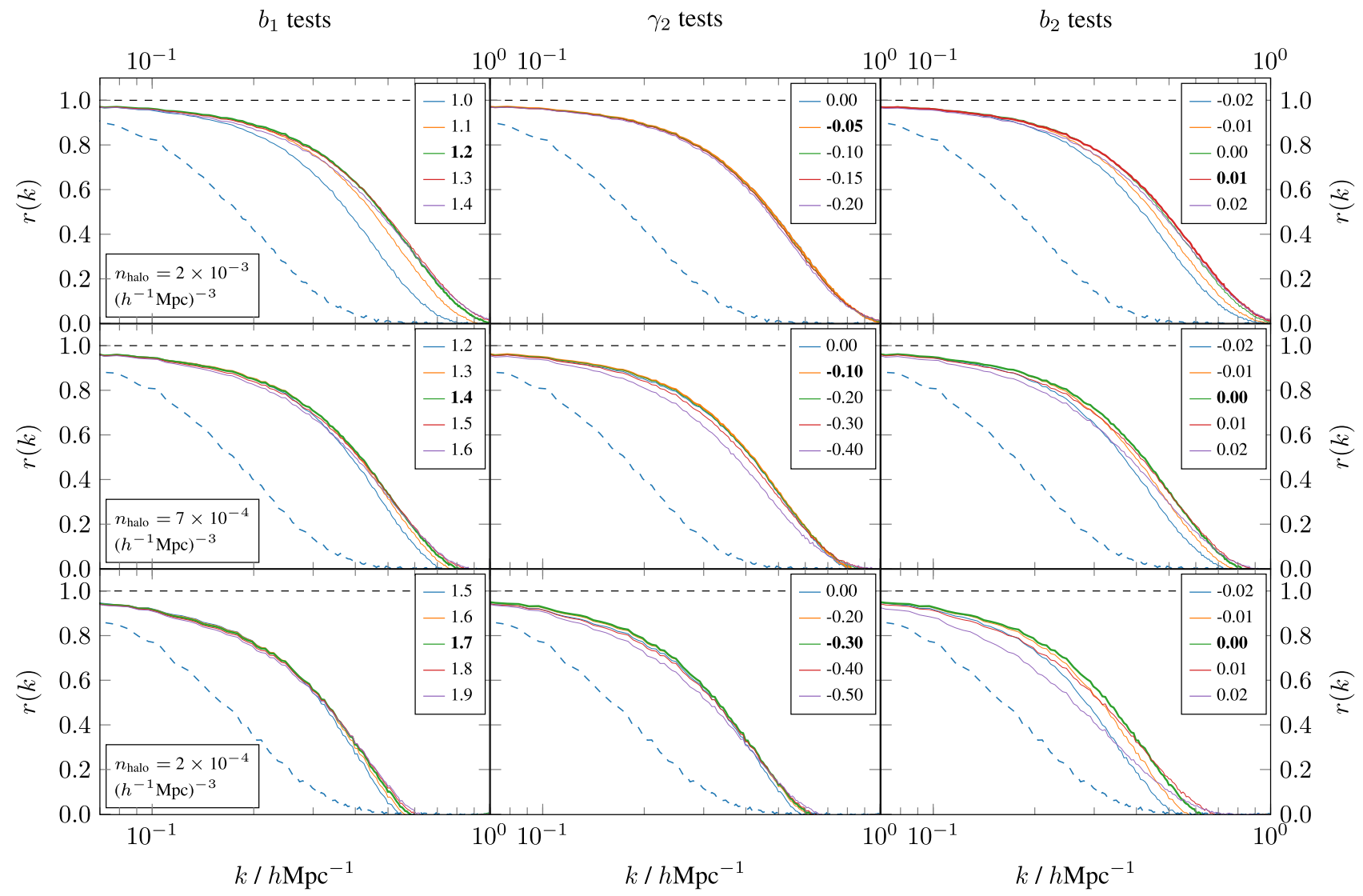

Figure 4. Correlation coefficients between the initial and final density fields $r_{\text {if }}$ (dashed), and between the initial and reconstructed density fields $r_{\text {ir }}$ (solid) for three halo samples with different number densities (each row corresponds to a different number density). Each column tests a different halo bias parameter: the linear bias, $b_{1}$ (left-hand column); the non-local bias, $\gamma_{2}$, using the optimal $b_{1}$ value (middle column); and the quadratic bias, $b_{2}$, using the optimal $b_{1}$ and $\gamma_{2}$ values (right-hand column). The optimal value in each panel is indicated by a thicker curve and a bold value in the legend.

reduce the largest values of $\delta_{\mathrm{h}}$ and therefore allow $b_{2}$ to be included, but this is beyond the scope of this work.

In general, the reconstruction performance varies little between the two types of tracers considered, however we find that HOD galaxies have a larger associated linear and non-local bias for a given number density. From the simulation, we measure $b_{1}=1.25,1.5$, and 2.0 for $n_{\text {galaxy }}=2 \times 10^{-3}, 7 \times 10^{-4}$, and $2 \times 10^{-4}\left(h^{-1} \mathrm{Mpc}\right)^{-3}$ respectively, and we find these values to be optimal for reconstruction in the three cases $\left(b_{1}=1.25\right.$ was not tested but $b_{1}=1.3$ was the best value chosen). The tests of non-local bias found $\gamma_{2} \approx-0.10$, -0.20 , and -0.60 to be optimal for reconstruction from the three corresponding number density distributions. We note that while equation (29) gives a poor estimate for $\gamma_{2}$, it need only be multiplied by a factor of 2 to give agreement with the galaxy reconstruction results.

\subsection{Recovery of the BAO peaks}

Having found the optimal bias values $\left(b_{1}, \gamma_{2}, b_{2}\right)$ for each tracer (halo and galaxy) catalogue and number density sample, we now assess the recovery of the BAO peaks using biased tracer reconstruction.

The left-hand panels of Fig. 6 show the correlation coefficients $r_{\text {if }}$ (blue) and $r_{\text {ir }}$ (orange) from halo (solid lines), galaxy (dashed lines) and matter (dotted lines) reconstruction for the three tracer number densities as before, decreasing from top to bottom. For matter reconstruction the curves are the same in all three rows. These plots show that tracer reconstruction generally performs worse than matter reconstruction, even for the highest number density used here, but increasing $n_{\text {tracer }}$ does bring $r_{\text {ir }}$ closer to the matter reconstruction case; it will be interesting to study the value of $n_{\text {tracer }}$ at which $r_{\text {ir }}$ for tracers and matter become very close. On the other hand, $r_{\text {if }}$ depends less sensitively on the tracer number density.

The panels in the right-hand column of Fig. 6 show the power spectra of the initial, non-linear halo, and reconstructed density fields in the form $\left(P / P_{\mathrm{nw}}\right)-1$, where $P_{\mathrm{nw}}$ comes from a simulation identical to that of the original, except that there are no BAO wiggles in the linear power spectrum used to generated the simulation initial conditions. Plotting this quantity allows us to clearly visualize the damping, due to non-linear structure formation, and the recovery, due to reconstruction, of the BAOs in the power spectrum. The black solid curve, which represents the BAO of the initial linear matter power spectrum, is identical in all three rows, whereas the blue curves, which represent the BAO peaks in the $z=0$ halo power spectrum, are dependent on the halo number density $n_{\text {halo }}$. The damping of the $\mathrm{BAO}$ wiggles is more severe when $n_{\text {halo }}$ is low, and the curves become very noisy, particularly in the lowest number density case. The loss of information from the initial conditions increases with the damping of the BAO wiggles, and this is more significant in the lower halo number density cases where $r_{\text {if }}$ drops off towards zero more rapidly. Similarly, the BAO wiggles are recovered to a great extent when the halo number density is greater, as would be expected from the left-hand panels. Note that we have 


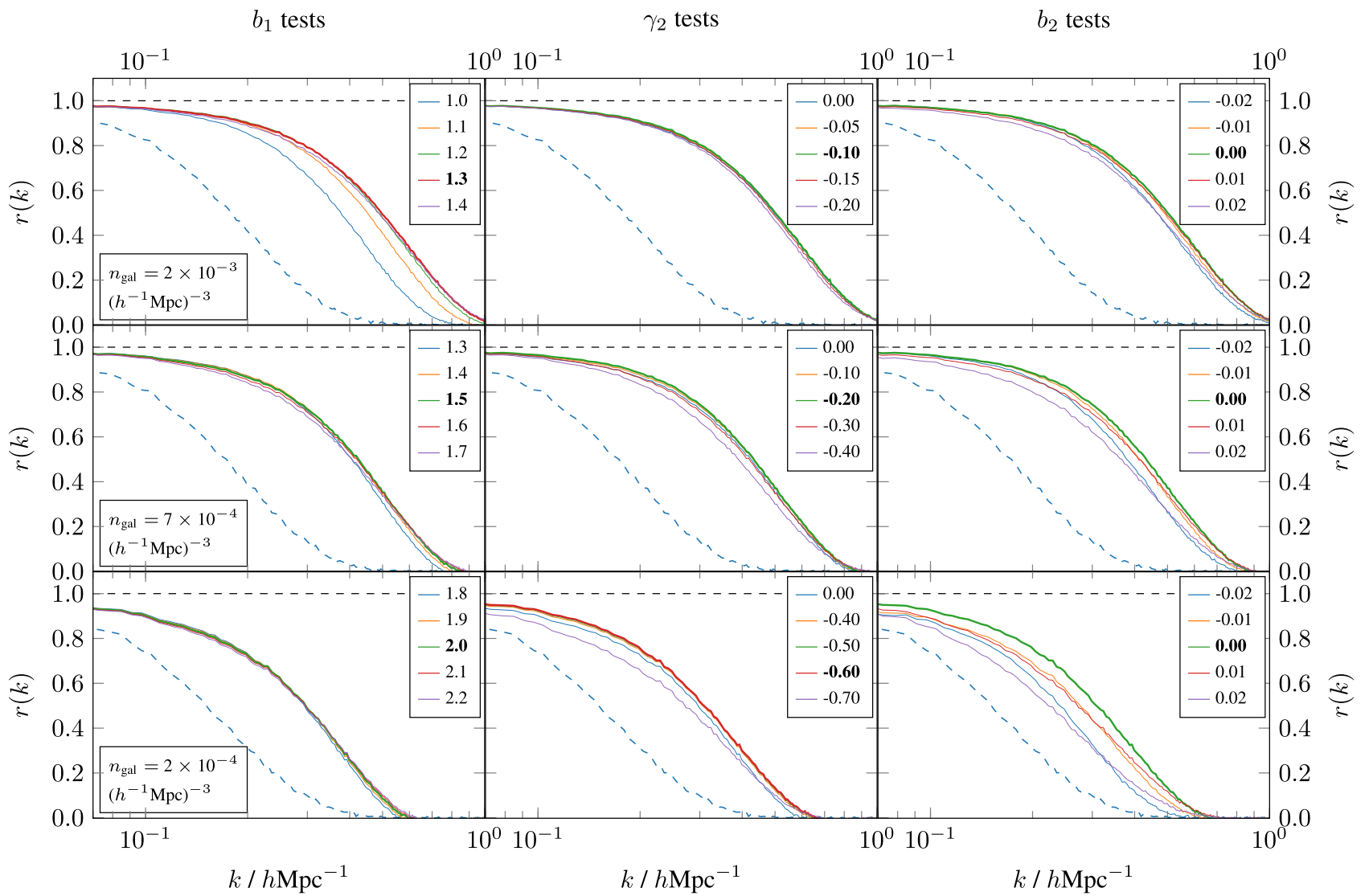

Figure 5. Same as Fig. 4, but now the reconstruction has been performed using HOD galaxy distributions which are built on the halo catalogues used for Fig. 4. Each row corresponds to a different galaxy number density which is the same as the halo number density in the corresponding row in Fig. 4.

Table 4. The $k_{80}, k_{50}$, and $k_{20}$ values corresponding to $r_{\text {ir }}$ found for halo and galaxy reconstruction with different halo number densities and different bias parameters.

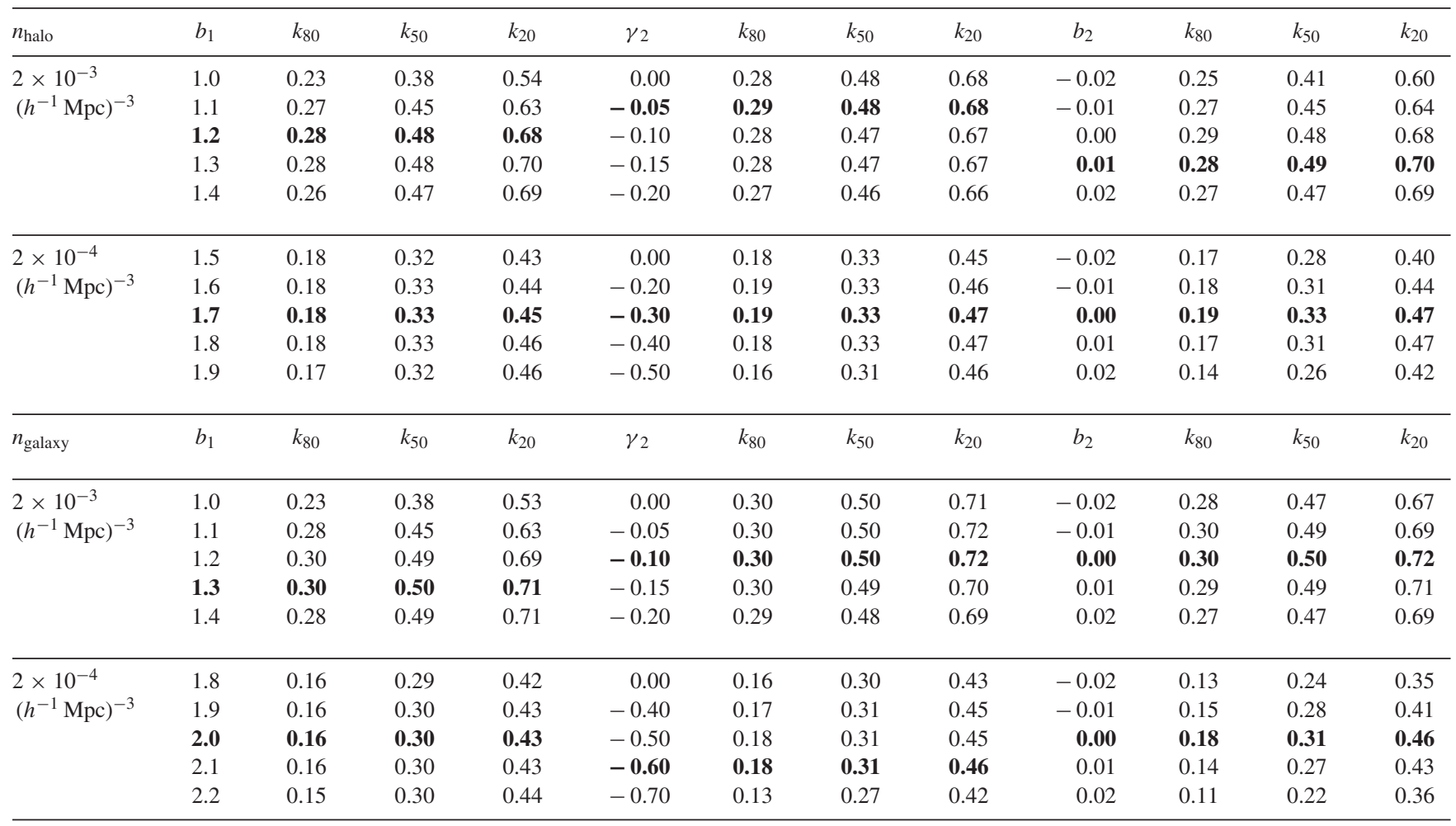




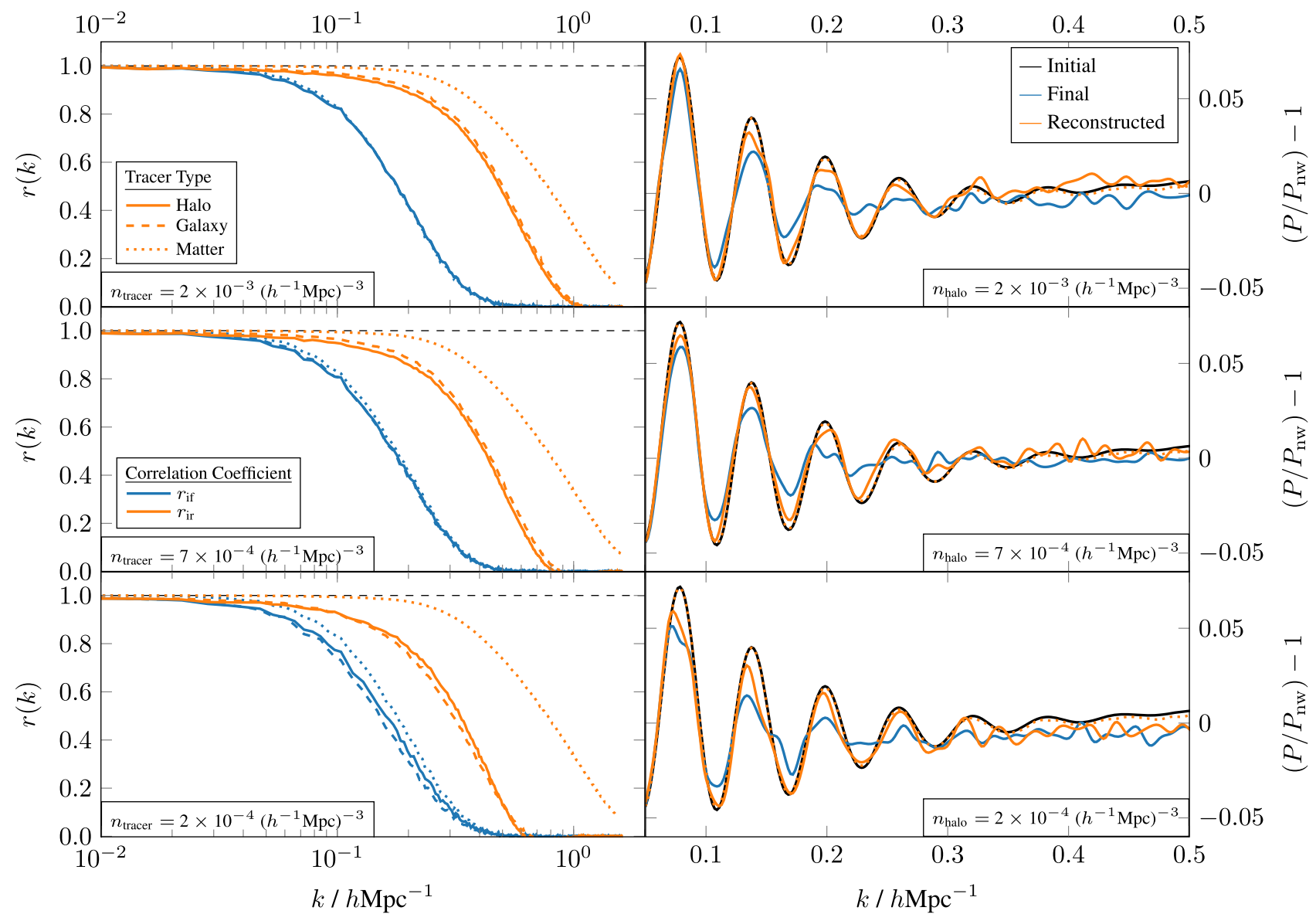

Figure 6. Left-hand column: correlation coefficients with the initial density distribution of the final $\left(r_{\mathrm{if}}\right.$; blue $)$ and reconstructed density $\left(r_{\mathrm{ir}}\right.$; orange) fields, for matter (dotted lines), haloes (solid lines), and HOD galaxies (dashed lines). The three panels show the reconstruction results for different tracer number densities, while the dotted lines (for matter reconstruction) are identical in all panels. Right-hand column: the BAO wiggles in the power spectrum plotted as $P(k) / P_{\text {nw }}(k)-1$, where $P(k)$ and $P_{\text {nw }}(k)$ are, respectively, the power spectra extracted from the full and from the no-wiggle simulations. It shows the power spectra ratio for the initial conditions (black solid lines), the final halo distribution (blue solid lines), and the reconstructed initial density from the dark matter (orange dotted lines) and halo (orange solid lines) distributions. For clarity, the right-hand panels do not show the result of the HOD galaxy reconstruction. All halo and galaxy reconstruction results are obtained using the optimal bias parameter values $\left(b_{1}, \gamma_{2}, b_{2}\right)$ from Figs 4 and 5.

omitted the galaxy power spectra due to the similarity of the cross correlations with haloes for all three number densities.

The BAO wiggles from the reconstructed density fields are shown in orange in the right-hand panels of Fig. 6, with dotted and solid lines representing respectively the results from dark matter and halo reconstruction. The dotted orange lines are the same in all three rows, and they show that dark matter reconstruction is capable of recovering the BAO peaks down to $k \approx 0.4 h \mathrm{Mpc}^{-1}$. Halo reconstruction does not perform as well, as expected, but for all three halo number densities, we still observe a substantial recovery of the BAO wiggles, e.g. compared with the blue curves, in the first four peaks, down to $k \approx 0.25 \mathrm{~h} \mathrm{Mpc}^{-1}$. The improvement is substantial for all halo number densities. Note that the orange and blue curves have been shifted vertically to align them with the black curve. This is because the same value of $b_{1}$ was used for both the wiggle and no-wiggle simulations, when in reality the measured values differ by roughly 1 per cent, and so taking the ratio of the $P(k)$ and $P_{\mathrm{nw}}(k)$ propagates this error to $\sim 2$ per cent. It is therefore appropriate to shift the curves to provide a clearer comparison.

To assess the competitiveness of our method, we can compare the enhancement of the BAO feature with results of other reconstruction approaches. The Yu et al. (2017) study represents a suitable comparison as they have applied their non-linear reconstruction procedure to similar populations of tracers and redshifts as us. For example, comparing our $n_{\text {halo }}=2 \times 10^{-3}\left(h^{-1} \mathrm{Mpc}\right)^{-3}$ results with the $n_{\text {halo }}=2.77 \times 10^{-3}\left(h^{-1} \mathrm{Mpc}\right)^{-3}$ there, we find that our method performs better in this case. In particular, there the reconstructed density field is approximately 95 per cent and 65 per cent correlated with the initial conditions at $k=0.1$ and $0.3 \mathrm{~h} \mathrm{Mpc}^{-1}$ respectively (see their fig. 2), whereas we find a correlation coefficient of 95 per cent and 80 per cent for the same $k$ values. We note, however, that this difference is likely due to the different density assignment schemes used - DTFE there and TSC here (as we have found above, using DTFE causes additional smoothing of the pre-reconstruction density field, which can downgrade the outcome of reconstruction even though it is not related to the reconstruction method itself).

\section{SUMMARY, DISCUSSION, AND CONCLUSIONS}

We have tested the non-linear density reconstruction method introduced by Shi et al. (2018), applying it to late-time halo and galaxy distributions, to study the potential of recovering BAO peaks 
from a tracer field, and how this depends on a number of factors including tracer type, tracer number density, mass assignment scheme, reconstruction grid resolution, and tracer bias parameters up to quadratic order. For this, we have developed an extension to the original Shi et al. (2018) method to incorporate non-local and non-linear tracer bias. These terms can be included naturally in the reconstruction equation - which is a non-linear PDE that takes the form of the Monge-Ampere equation - by changing the coefficients and source terms of the equation. The original numerical algorithm still works efficiently when applied to the new equation.

Our results confirm that, as expected, tracer number density plays an important role in determining the performance of reconstruction (which we assess by calculating the correlation coefficient, $r_{\mathrm{ir}}$, between the initial and reconstructed density fields), with higher number density tracers giving larger $r_{\text {ir }}$ values. The mass assignment scheme used to calculate the tracer density at each position is another important factor for reconstruction performance, with TSC faring significantly better than DTFE and slightly better than CIC for all tracer number densities used. Using a sufficiently high-resolution computational grid for reconstruction is also crucial, and we find that once the grid cell size decreases to $\sim 1-2 h^{-1} \mathrm{Mpc}$ the results converge for all tracer number densities studied here. Reconstruction from HOD galaxy and halo distributions with the same number density gives quite similar results.

Of the three bias parameters studied in this work, the linear tracer bias, $b_{1}$, is by far the most important. For high tracer number densities, the reconstruction performance depends sensitively on it, while this dependence is much weaker for low tracer number densities. In all cases, we find that the linear bias parameter measured in the simulation by comparing the clustering of dark matter and halo/galaxy distributions works best, but using larger (by up to 0.2) values does not affect the reconstruction significantly. For TSC mass assignment, we find that the non-local bias parameter $\gamma_{2}$ predicted by perturbation theory is close to the values that give rise to the best reconstruction result, but this is not the case when DTFE mass assignment is used, which is another reason why we use TSC in the bias analysis. Including non-local bias, however, only marginally improves $r_{\text {ir }}$, with the largest improvement found for the lowest number density sample, for which the optimal $\left|\gamma_{2}\right|$ value is also the largest. Finally, the non-linear bias at quadratic order, $b_{2}$, if nonzero, can lead to poorer reconstruction, because our reconstruction method calculates the displacement field on all scales, while the non-linear bias does not work on small scales where the density field can become large.

These results are confirmed by visually inspecting the recovery of the BAO peaks, as shown in the right-hand panels of Fig. 6. We can see there that, when applied to halo reconstruction using the best-fitting bias parameters, our method substantially improves the recovery of the first few BAO peaks compared with the case of no reconstruction, down to $k \sim 0.25 h \mathrm{Mpc}^{-1}$.

For all the tracer reconstruction results shown here, the tracer density field, $\delta_{\mathrm{h}}$, is calculated by treating the tracers as particles of equal mass, which is a simplified assumption. For example, some haloes are more massive (e.g. $>10^{15} h^{-1} \mathrm{M}_{\odot}$ ) than others (e.g. $<10^{12} h^{-1} M_{\odot}$ ). Naturally, more massive haloes contain more matter, implying that the non-linear dark matter field may be more reliably constructed using a mass-weighted halo number density field. To verify this, we have also carried out halo reconstruction tests in which $\delta_{\mathrm{h}}$ is calculated using the actual masses of the haloes. However, this approach leads to a poorer reconstruction, with the resulting $r_{\text {ir }}$ being smaller than the ones seen in Fig. 3, in particular for the high halo number density case. This happens regardless of the value of $b_{1}$ used, and it could be because the simple mass-weighting scheme above gives too little weight to low-mass haloes, which are important tracers of the underlying matter field. This therefore indicates a more sophisticated weight scheme may be required. We leave an investigation on this to future work.

As mentioned above, in principle our method for biased tracer reconstruction can be straightforwardly generalized to higher order bias terms. For example, the non-local bias at cubic order can be similarly expressed in terms of derivatives of the displacement potential, $\theta$, amounting to a further change of various coefficients in the reconstruction equation (13). However, we have decided not to pursue this line of research, given that the effect of including bias terms up to the quadratic order is already small.

As the first attempt to add more reality to the reconstruction method of Shi et al. (2018), in this work we have only considered a few simple cases of tracer reconstruction. In order to be able to apply the method to observational data, such as galaxy catalogues, a few important factors need to be taken into account. First, while the tests in this paper have all been done in a cubic box for a fixed snapshot $(z=0)$, both the spatial and the redshift distributions of galaxies in real observations are more complicated. For example, observed galaxy catalogues are usually in a light-cone rather than a box, and certain regions of the field of view are masked with no data collected; for reconstruction, we will need to embed the light-cone into a cubic box, adding a density field (e.g. zero, random, or uniform) outside the light-cone ensuring periodic boundary conditions. Second, real galaxy catalogues may suffer from incompleteness issues which can be caused by observing conditions, redshift failures, fibre collisions, etc., and care must be taken to deal with this or make corrections. Third, while we have used constant bias values in this study, for observed galaxy catalogues covering a significant redshift interval the bias parameters do evolve, and this should be taken into account as well. Fourth, in this study we have not considered the redshift space distortions of galaxy line-of-sight (los) distances, but in reality only the redshifts of galaxies are measured, whose relation with the los distances are complicated due to coherent and virialized galaxy motions (see e.g. Zhu, Yu \& Pen 2018; Hada \& Eisenstein 2018, for some recent studies of reconstruction from redshift space). It will be interesting to extend the reconstruction method used here to include redshift space distortions. It is also useful to apply the method to different tracer types (bright galaxies, luminous red galaxies, emission line galaxies, quasars, etc.), which cover different redshift ranges and have different bias properties. In order to verify its accuracy, it is also important to test the final pipeline using some realistic mock galaxy catalogues (e.g. Smith et al. 2017). We leave these interesting developments to future works.

\section{ACKNOWLEDGEMENTS}

We thank Xin Wang and Hong-Ming Zhu for helpful discussions during this project and the anonymous referee for their insightful comments. JB and BL are supported by the European Research Council (ERC-StG-716532-PUNCA), and BL and MC are supported by the STFC through grant ST/P000541/1. This work used the DiRAC Data Centric system at Durham University, operated by the Institute for Computational Cosmology on behalf of the STFC DiRAC HPC Facility (www.dirac.ac.uk). This equipment was funded by BIS National E-infrastructure capital grant ST/K00042X/1, STFC capital grant ST/H008519/1, and STFC 
DiRAC Operations grant ST/K003267/1 and Durham University. DiRAC is part of the National E-Infrastructure.

\section{REFERENCES}

Aghamousa A. et al., 2016, preprint (arXiv:1611.00036)

Alam S. et al., 2017, MNRAS, 470, 2617

Alonso D., 2012, preprint (arXiv:1210.1833)

Aubourg É. et al., 2015, Phys. Rev. D, 92, 123516

Behroozi P. S., Wechsler R. H., Wu H.-Y., 2013, ApJ, 762, 109

Berlind A. A., Weinberg D. H., 2002, ApJ, 575, 587

Brenier Y., Frisch U., Hénon M., Loeper G., Matarrese S., Mohayaee R., Sobolevskiǔ A., 2003, MNRAS, 346, 501

Cautun M. C., van de Weygaert R., 2011, Astrophysics Source Code Library (ascl:1105.003)

Chan K. C., Scoccimarro R., Sheth R. K., 2012, Phys. Rev. D, 85, 083509

Cole S. et al., 2005, MNRAS, 362, 505

Crocce M., Pueblas S., Scoccimarro R., 2006, MNRAS, 373, 369

Croft R. A. C., Gaztanaga E., 1997, MNRAS, 285, 793

Desjacques V., Jeong D., Schmidt F., 2018, Phys. Rep., 733, 1

Eisenstein D. J. et al., 2005, ApJ, 633, 560

Eisenstein D. J., Hu W., 1998, ApJ, 496, 605

Eisenstein D. J., Seo H.-J., Sirko E., Spergel D. N., 2007, ApJ, 664, 675

Frisch U., Matarrese S., Mohayaee R., Sobolevski A., 2002, Nature, 417, 260

Fry J. N., Gaztanaga E., 1993, ApJ, 413, 447

Hada R., Eisenstein D. J., 2018, MNRAS, 478, 1866

Hockney R. W., Eastwood J. W., 1988, Computer Simulation using Particles. Institute of Physics Publishing, Bristol

Ivezić Ž. et al., 2008, preprint (arXiv:0805.2366)

Jasche J., Lavaux G., 2018, preprint (arXiv:1806.11117)

Jasche J., Wandelt B. D., 2013, MNRAS, 432, 894

Kitaura F. S., Enßlin T. A., 2008, MNRAS, 389, 497

Laureijs R. et al., 2011, preprint (arXiv:1110.3193)
Lavaux G., 2016, MNRAS, 457, 172

Li B., Zhao G.-B., Teyssier R., Koyama K., 2012, J. Cosmol. Astropart. Phys., 2012, 051

Mohayaee R., Mathis H., Colombi S., Silk J., 2006, MNRAS, 365, 939

Padmanabhan N., Xu X., Eisenstein D. J., Scalzo R., Cuesta A. J., Mehta K. T., Kazin E., 2012, MNRAS, 427, 2132

Peebles P. J. E., 1989, ApJ, 344, L53

Press W. H., Teukolsky S. A., Vetterling W. T., Flannery B. P., 2007, Numerical Recipes: The Art of Scientific Computing, 3rd edn. Cambridge Univ. Press, Cambridge

Schaap W. E., van de Weygaert R., 2000, A\&A, 363, L29

Schmittfull M., Baldauf T., Zaldarriaga M., 2017, Phys. Rev. D, 96, 023505

Scoccimarro R., 1998, MNRAS, 299, 1097

Shi Y., Cautun M., Li B., 2018, Phys. Rev. D, 97, 023505

Smith A., Cole S., Baugh C., Zheng Z., Angulo R., Norberg P., Zehavi I., 2017, MNRAS, 470, 4646

Teyssier R., 2002, A\&A, 385, 337

Vargas-Magaña M., Ho S., Fromenteau S., Cuesta A. J., 2017, MNRAS, 467, 2331

Wang X., Pen U.-L., 2018

Wang H., Mo H. J., Yang X., Jing Y. P., Lin W. P., 2014, ApJ, 794, 94

Wang X., Yu H.-R., Zhu H.-M., Yu Y., Pan Q., Pen U.-L., 2017, ApJ, 841 , L29

Weinberg D. H., 1992, MNRAS, 254, 315

Yu Y., Zhu H.-M., Pen U.-L., 2017, ApJ, 847, 110

Zheng Z. et al., 2005, ApJ, 633, 791

Zheng Z., Coil A. L., Zehavi I., 2007, ApJ, 667, 760

Zhu H.-M., Yu Y., Pen U.-L., Chen X., Yu H.-R., 2017, Phys. Rev. D, 96, 123502

Zhu H.-M., Yu Y., Pen U.-L., 2018, Phys. Rev., D97, 043502

This paper has been typeset from a $\mathrm{T}_{\mathrm{E}} \mathrm{X} / \mathrm{L} \mathrm{T} \mathrm{E} \mathrm{X}$ file prepared by the author. 